\title{
COLOCACIÓN DE IMPLANTES DE OSEOINTEGRACIÓN EN MANDÍBULAS CON REABSORCIÓN SEVERA UTILIZANDO LA TÉCNICA “TENT POLE” SERIE DE CASOS
}

\author{
Camilo Eslavai, Paolo Veronai, Elías Franco ${ }^{*}$ \\ ${ }^{1}$ Especialista en Cirugía Oral y Maxilofacial del Hospital Militar Central. Universidad Militar Nueva Granada \\ ${ }^{2}$ Residente en Cirugía Oral y Maxilofacial del Hospital Militar Central. Universidad Militar Nueva Granada \\ *Correspondencia: elias.pretto@gmail.com.
}

Recibido: Marzo 25 de 2013 Aceptado: Julio 7 de 2013

\section{Resumen}

La rehabilitación protésica mandibular implanto-soportada actualmente es una excelente opción de tratamiento para pacientes edéntulos, ya que brinda adecuada estabilidad y mejor acceso para la higiene. La reabsorción del reborde alveolar mandibular ocurre en sentido vertical y horizontal. Muchos de estos pacientes presentan alturas alveolares que oscilan entre los $3 \mathrm{~mm}$ y $8 \mathrm{~mm}$, aumentando el brazo de palanca de la prótesis con respecto al soporte mandibular.

La opción de aumentar el reborde alveolar en altura y grosor mediante la técnica "Tent Pole", evita futuras complicaciones como fracturas patológicas que sin duda complicarían el manejo. Utilizando este método no solo se logra un aumento de grosor y altura alveolar, sino que también evita la contaminación de los injertos que ocurre a través del abordaje intraoral (1-4). A continuación, presentamos una serie de tres casos utilizando esta técnica.

Palabras clave: Tent Pole, atrofia mandibular, implantes de oseointegración

\section{OSSEOINTEGRATED IMPLANTS PLACEMENT IN SEVERLY RESORBED MANDIBLES USING THE “TENT POLE” TECHNIQUE. CASE SERIES}

\begin{abstract}
Mandibular rehabilitation with implant-supported prostheses is now an excellent treatment option for edentulous patients because it provides increased stability and improved oral hygiene. Mandibular alveolar resorption occurs in vertical and horizontal vectors. There are patients presenting mandibular alveolar heights between 3 and $8 \mathrm{~mm}$ which unfavorably increases the prosthetic level arm.

The option to increase the alveolar height and thickness before implant placement avoids complications like pathological fractures which compromises the results. By using the "Tent Pole" technique the surgeon achieves an increase of alveolar width and height, and by the way preventing contamination of the grafts through communication with the oral cavity, (1-4). We present a series of three cases using this technique.
\end{abstract}

Key words: Tent Pole, osseointegrated implants, resorbed mandible. 


\title{
COLOCAÇÃO DE IMPLANTES OSSEOINTEGRAÇÃO IN JAWS COM TÉCNICA DE ABSORÇÃO INTENSA UTILIZANDO “TENT POLE”. SERIES CASE
}

\section{Resumo}

\begin{abstract}
Reabilitação mandibular implanto-suportada próteses atualmente é uma excelente opção de tratamento para pacientes desdentados totais, uma vez que proporciona uma boa estabilidade e um melhor acesso para a higiene. O rebordo alveolar mandibular reabsorção ocorre verticalmente e horizontalmente. Muitos destes pacientes têm alturas alveolares que variam de 3 a $8 \mathrm{~mm}$, aumentando o braço de alavanca da prótese em relação ao suporte inferior.
\end{abstract}

A opção de aumentar a altura e espessura do rebordo alveolar utilizando a técnica de "pau da barraca", evitar complicações futuras, como fraturas patológicas certamente complicar a gestão. Usando este método, não só alcança um aumento da espessura e altura alveolar, mas também evita a contaminação dos enxertos, que ocorre através da via intra-oral (1-4). Aqui estão uma série de três casos, utilizando esta técnica.

Palavras chave: Tent Pole, atrofia mandibular, implantes osseointegração.

\section{Introducción}

La rehabilitación implantosoportada de los maxilares, constituye una forma predecible de tratar el edentulismo cuando se utiliza la técnica adecuada en una situación clínica óptima. Sin embargo, en el caso de pacientes con mandíbulas severamente reabsorbidas $(<6 \mathrm{~mm}$ de altura), este método podría ser difícil sino imposible de realizar, por el riesgo elevado de producir fracturas patológicas, dehiscencias, infecciones y defectos de continuidad (1-5). La reabsorción mandibular severa se observa en pacientes geriátricos con pérdida dental de larga evolución, pacientes con displasia ectodérmica, pacientes con secuela de trauma facial con pérdida extensa de segmentos dentoalveolares, que convierten la colocación de implantes de oseointegración en un procedimiento técnicamente difícil de realizar (6). Estos pacientes deben ser manejados con técnicas de reconstrucción mandibular de manera previa a la colocación de implantes de oseointegración. A través del tiempo, esta situación ha sido manejada mediante diversas técnicas quirúrgicas, pero ninguna ha conseguido resultados óptimos a largo plazo en términos de estabilidad ni función protésica en este grupo de pacientes.

Existen series de casos que reportan la utilización de injertos autólogos costales para la reconstrucción de mandíbulas con reabsorción severa $(7,8)$. La técnica consistía en tomar dos costillas del paciente, una de las cuales se colocaba sobre la superficie crestal del reborde mandibular a través de un abordaje intraoral, y la otra costilla se particularizaba para colocarla en las superficies vestibular y lingual. Se reportó un $15 \%$ de casos con dehiscencias y subsecuente pérdida del injerto,aquellos casos que no presentaron dehiscencia, mostraron reabsorción completa del injerto al término de 18 meses. Las causas del fracaso de los injertos fueron principalmente el cierre a tensión de los abordajes intraorales, dehiscencia, la hipovascularidad de la mucosa del reborde alveolar y la contracción del tejido sobre el injerto.

Otros autores sugirieron osteotomías alveolares mandibulares para aumentar la altura de la mandíbula reabsorbida. Stoelinga (9) propuso una osteotomía horizontal alveolar en la región comprendida entre las entradas de los nervios mentonianos y colocar un injerto en bloque entre el segmento coronal del fragmento osteotomizado y el basal, técnica a la que llamó osteotomía en "sándwich". Harle (10) diseño la osteotomía "en viscera" que a diferencia de la de Stoelinga incluía un corte vertical lingual que dividía el hueso mandibular y se conectaba en el sector anterior con el corte horizontal en la región comprendida entre la entrada de los nervios mentonianos. Esta técnica permite desplazar el segmento lingual hacia coronal y colocar los injertos óseos interposicionales para el aumento de la altura del reborde. Estos procedimientos acarrearon muchas complicaciones, entre ellas, dehiscencias, infecciones, 
disestesia, anestesia, desplazamiento de segmentos óseos, fracturas patológicas y pérdida de los injertos.

Otras técnicas han incluido injertos óseos en bloque tipo "onlay", injertos óseos autólogos particularizados, distracción osteogénica, mallas de titanio, y la combinación de todas $(11,12)$. El problema principal de las técnicas de aumento de reborde alveolar es la comunicación del injerto con el medio oral debido a la alta tasa de dehiscencias. Louis y cols (11) en su estudio registraron una tasa de dehiscencia de 52,7\% utilizando la malla de titanio como matriz contenedora del injerto. Block y Degen (13) sugirieron la colocación de un aloinjerto de hueso humano particularizado mineralizado sin la colocación de malla, mediante una técnica de tunelización subperióstica. No se registraron dehiscencias, sin embargo, se observó migración apical del injerto, resultado de la contracción natural de la mucosa oral.

El objetivo principal de las técnicas de aumento de reborde alveolar es proveer un lecho adecuado para el soporte protésico. La evidencia demuestra que los injertos óseos sufren un período normal de remodelación una vez colocados, pero la reabsorción se perpetúa cuando no existe una carga funcional en la zona injertada. Eyrich y cols., en su serie de casos, afirma que el aumento del reborde mandibular edéntulo mediante injertos óseos es un procedimiento más estable, cuando se colocan los implantes de oseointegración de manera simultánea (14).

Marx y cols describieron la técnica "Tent Pole" para la reconstrucción de mandíbulas de pacientes con reabsorción severa en el año 2002 (1). Consiste en exponer la zona anterior mandibular a través de un abordaje submental. La técnica combina la colocación de implantes de oseointegración e injerto autólogo corticomedular de cresta ilíaca en un solo tiempo quirúrgico. Los implantes sirven de pilares para mantener el espacio subperióstico y el efecto de "tienda" evitando las consecuencias negativas de la contracción tisular postoperatoria. Es una técnica que evita contacto con el medio intraoral y reduce en gran medida la reabsorción del injerto gracias a la expansión tisular obtenida.

\section{Técnica quirúrgica}

La técnica quirúrgica involucra la utilización de implantes dentales de oseointegración en un procedimiento simultáneo a la colocación de injerto óseo autólogo. Es un procedimiento que puede ser dividido en dos partes: La primera parte conlleva la creación de la matriz expandida de tejido blando: Previa asepsia y antisepsia, se realiza marcación con azul de metileno para abordaje submental, infiltración local anestésica con vasoconstrictor. Con la técnica se pretende abordar el borde inferior mandibular en la región comprendida entre los forámenes mentonianos. Se realiza incisión del plano cutáneo y disección por planos hacia el borde inferior mandibular. Se diseca subperiósticamente solo en las superficies crestal y bucal; el borde inferior y la cara lingual de la mandíbula se mantienen intactos para asegurar el aporte vascular al hueso.

Se recomienda extender la disección hacia el límite anterior del cuerpo mandibular, incluyendo el trígono retromolar y la rama ascendente de la mandíbula de manera bilateral; esto asegura conseguir adecuada expansión del tejido. No es necesario realizar transposición del nervio mentoniano, éste generalmente se ubica en la región crestal y posterior (zona de segundo molar) en mandíbulas con reabsorción severa, por lo que no interferirá con el procedimiento.

La segunda parte de la técnica implica el mantenimiento del tejido expandido. Ésto se realiza colocando implantes dentales convencionales que también funcionarán como retenedores protésicos una vez se encuentren oseointegrados. Marx y cols (1) sugieren colocar entre cuatro a seis implantes de $4.0 \mathrm{~mm}$ de diámetro por 15 $\mathrm{mm}$ de longitud, ubicándolos en el centro del reborde reabsorbido. Los implantes son dirigidos hacia el borde inferior hasta conseguir estabilidad primaria. Como la altura alveolar en estos pacientes oscila entre 4 y $6 \mathrm{~mm}$, es evidente que la longitud remanente de los implantes se proyectará por encima de la cresta ósea. Se debe mantener espacio de $1 \mathrm{~cm}$ entre los implantes. El primero de los implantes se coloca a una distancia de 5 $\mathrm{mm}$ desde el centro de éste, anterior a la emergencia del nervio mentoniano, el resto de los implantes debe colocarse manteniendo el paralelismo. Los implantes paralelos sirven como postes para mantener la matriz creada mediante la disección subperióstica, de ahí el término "tent pole", cuya traducción al español es "poste para tienda".

Mantener suspendida la matriz de tejido blando es clave para evitar la contracción del tejido sobre el injerto, lo que induce reabsorción. El injerto óseo puede ser tomado de la cresta ilíaca anterior o posterior, dependiendo del volumen de hueso requerido. Luego de obtenido y particularizado, éste se coloca y se compacta 
desde el sector posterior hacia el anterior. En el sector anterior el injerto se compacta alrededor y por encima de los implantes.

La técnica puede ser combinada con la aplicación de plasma rico en plaquetas para incrementar el potencial osteogénico y osteoconductivo del injerto (1). Se realiza cierre por planos de todo el tejido blando excepto el periostio, el cual forma parte de la matriz expandida. Los tejidos intraorales se encuentran expandidos, pero no han sido perforados. Después de la segunda semana, se indica la utilización de prótesis provisional, y los implantes son descubiertos a los 3 meses postoperatorios para continuar con el manejo protésico.

\section{Caso clínico \# 1}

Paciente femenina de 60 años de edad con antecedente de osteoporosis y esclerodermia quien fue remitida al servicio de cirugía oral y maxilofacial para colocación de implantes de oseointegración para rehabilitación implantosoportada en agosto de 2008. Se realizan las interconsultas a reumatología y endocrinología para el control de sus comorbilidades. En el momento del examen clínico facial se observa disminución de la dimensión vertical facial anterior, colapso labial, y ptosis de comisuras labiales (figura1). La Paciente es llevada a salas de cirugía para colocación de cuatro implantes de oseointegración según la planificación clínica y tomográfica (figura 2). Se colocan 4 implantes de oseointegración de 3,75 mm de diámetro por 13 $\mathrm{mm}$ de longitud (figura 3). La paciente culmina su tratamiento quirúrgico y de rehabilitación con ganancia ósea evidente en las imágenes de control postoperatorio en junio de 2009 (figura 4).

\section{Caso Clínico \# 2}

Paciente masculino de 24 años de edad con antecedente de herida de arma de fuego autoinfligida en la región facial en febrero de 2003. Después del manejo inicial, en mayo de 2003 es sometido a cirugía reconstructiva mandibular con colgajo libre de peroné por parte de cirugía plástica. Posteriormente es llevado a salas de cirugía en múltiples ocasiones para remodelación de la piel del colgajo, reconstrucción nasal, injerto de cresta ilíaca en mandíbula, cirugías de revisión de cicatriz de labio, colocación de conformadores nasales. Posterior a los procedimientos de cirugía plástica es remitido a nuestro servicio para colocación de implantes de oseointegración, para el examen clínico facial se evidencia disminución del tercio inferior en altura y colapso del tercio medio, pobre proyección anteroposterior del tercio medio e inferior. En el examen intraoral se evidencia pérdida de segmento alveolar anterior superior y reborde inferior (colgajo libre de peroné), insuficiente en

Revisión de la literatura
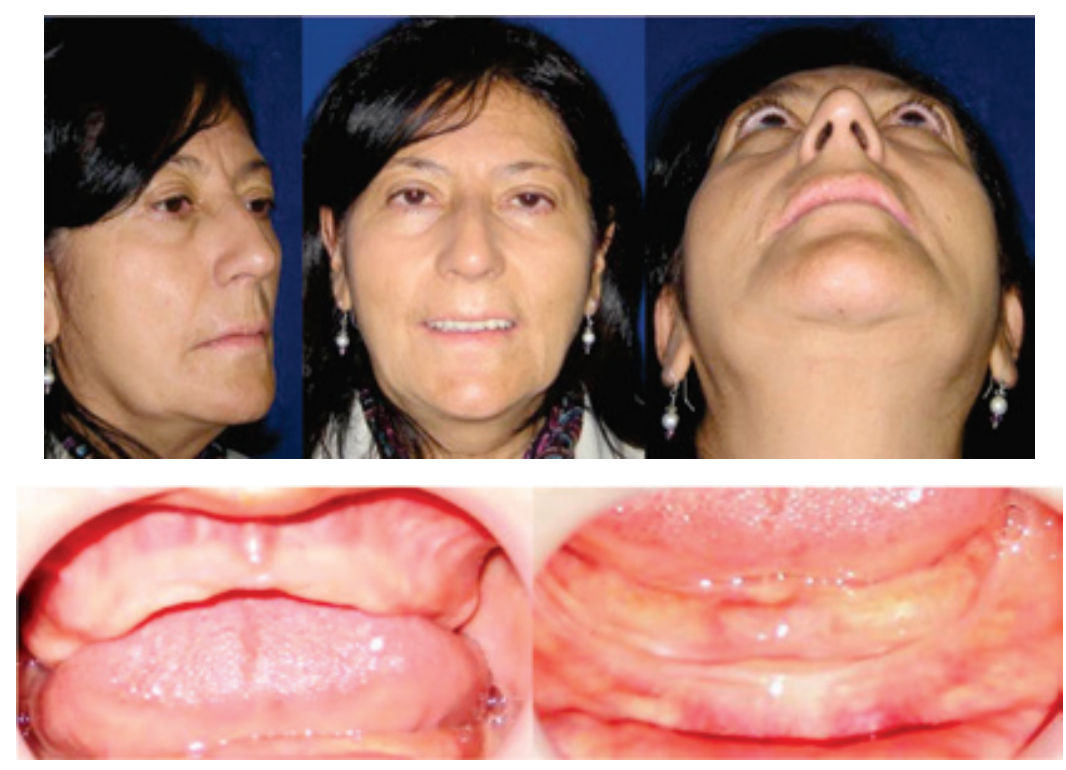

Figura 1. Caso Clínico \#1. Dimensión vertical disminuida. Paciente portadora de prótesis mucosoportada superior. Reborde mandibular con reabsorción extensa. 
COLOCACIÓN DE IMPLANTES DE OSEOINTEGRACIÓN EN MANDÍBULAS CON REABSORCIÓN SEVERA UTILIZANDO LA TÉCNICA “TENT POLE". SERIE DE CASOS

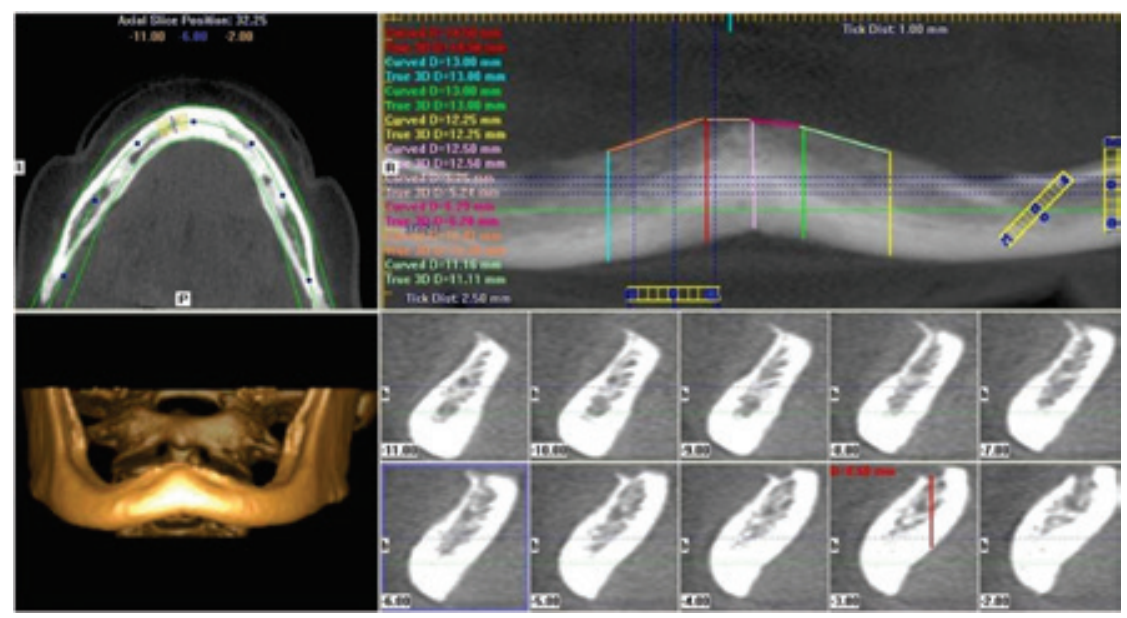

Figura 2. Caso Clínico \#1. Planificación Tomográfica para determinar las zonas de inserción de los implantes de oseointegración en la zona anterior mandibular.

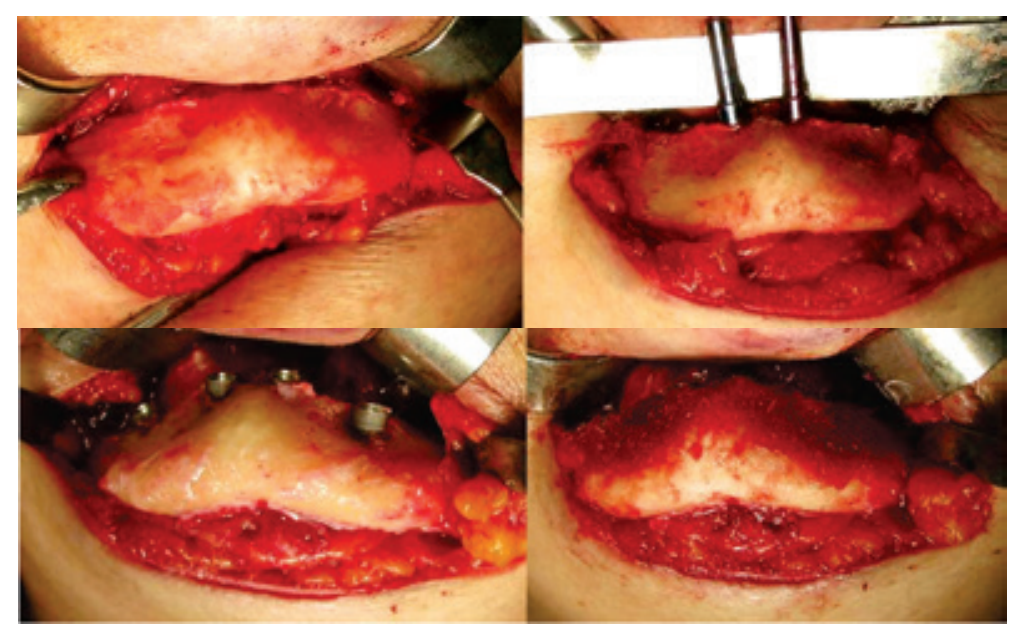

Figura 3. Colocación de cuatro implantes de oseointegración en el sector anterior con injerto óseo autólogo corticomedular de cresta ilíaca anterior.

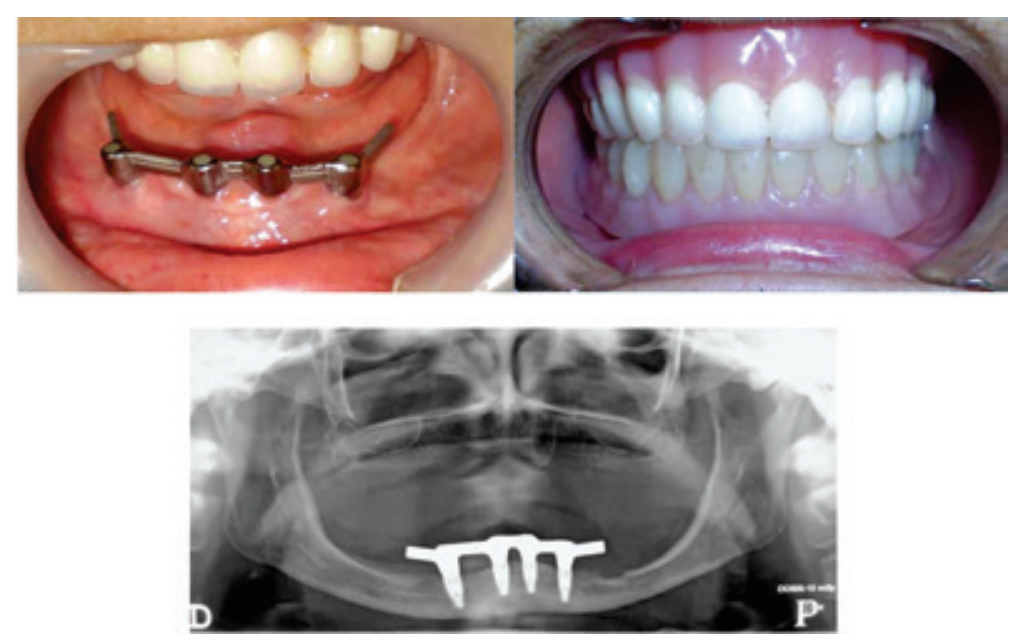

Figura 4. Postoperatorio imagenológico y clínico. Aumento del reborde mandibular y del volumen submucoso. Aspecto final de la rehabilitación protésica. 
altura para colocación de implantes de oseointegración (figura 5). En imágenes de diagnóstico se corrobora la altura insuficiente del colgajo de peroné (figura 6).

El paciente es llevado a cirugía en el mes de febrero de 2009, bajo anestesia general y mediante la técnica "Tent Pole" se colocan 6 implantes de oseointegración de 5,5 $\mathrm{mm}$ de diámetro por $15 \mathrm{~mm}$ de longitud, también es colocado un injerto corticomedular de cresta iliaca anterior y membrana de colágeno para regeneración ósea guiada (figura 7).

En marzo de 2009 el paciente ingresa a control postquirúrgico. En las radiografías los implantes de oseointegración se observan sin alteraciones, con un aumento de la altura del colgajo microvascular de peroné (figura 8).
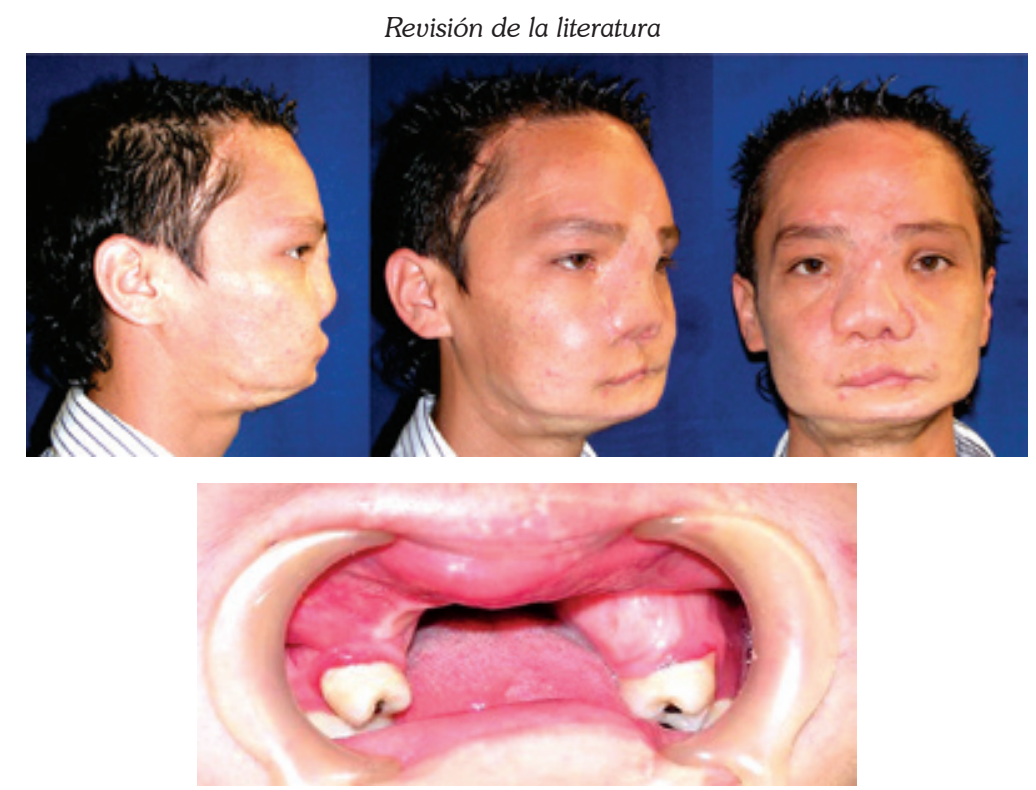

Figura 5. Caso Clínico \#2. Imágenes faciales e intraorales prequirúrgicas. Pérdida de dimensión vertical inferior, pobre proyección del tercio medio. Pérdida de segmento premaxilar y reborde inferior atrófico.

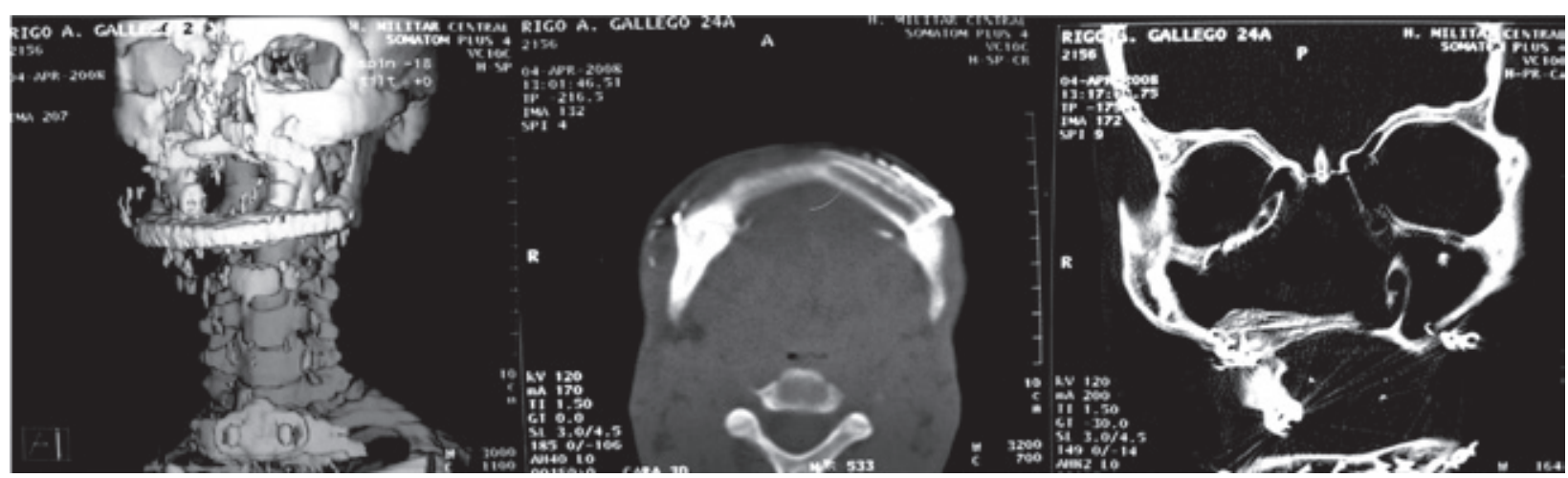

Figura 6. Tomografía Simple con Reconstrucción Tridimensional. Ausencia de segmento premaxilar, colgajo libre de peroné con altura remanente tomográfica de $6 \mathrm{~mm}$. Pérdida de soporte de tejidos blandos. 

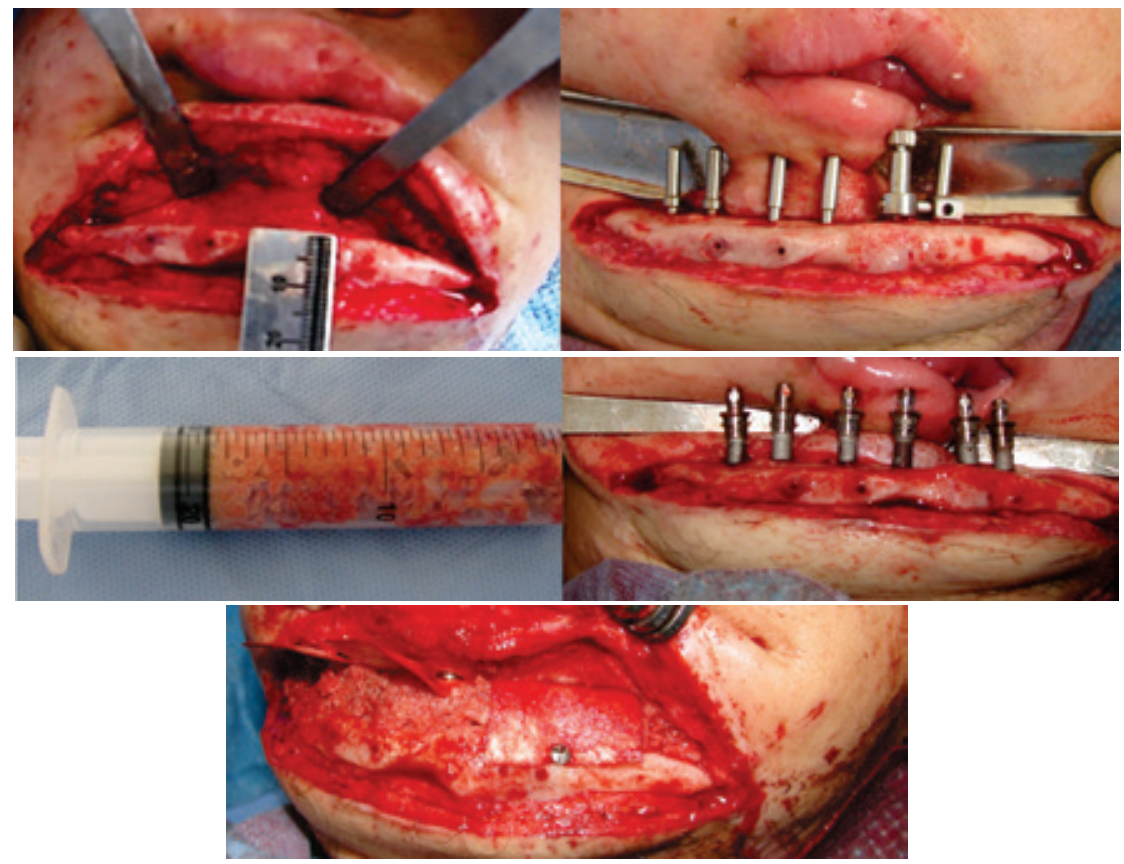

Figura 7. Secuencia Quirúrgica. Frente a una altura máxima de $9 \mathrm{~mm}$ se colocan 6 implantes de oseointegración. Se corrobora paralelismo y se toma injerto de cresta ilíaca anterior para el aumento del colgajo microvascular de peroné mediante regeneración ósea guiada.
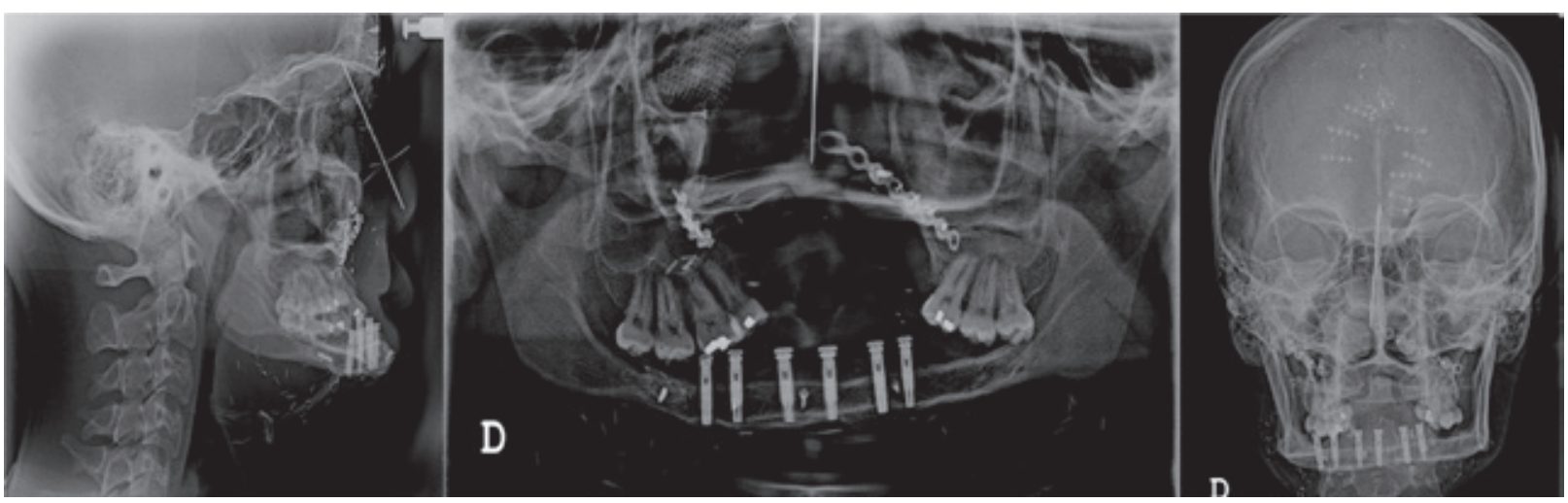

Figura 8. Control Radiográfico postoperatorio marzo 2009. Aumento del volumen óseo radiográfico.

\section{Caso Clínico \#3}

Paciente femenina de 59 años de edad con antecedente de síndrome de Ehler Danlos, osteoporosis, fractura patológica de cuerpo mandibular izquierdo posterior a exodoncia de resto radicular extrainstitucional en marzo de 2010. La fractura patológica fue manejada mediante reducción y colocación de placa de reconstrucción. Es remitida a nuestro servicio para la colocación de implantes de oseointegración. Al examen clínico se evi- dencia disminución de la dimensión vertical, ptosis de comisuras labiales y pobre proyección anteroposterior del tercio medio (figura 9). Se realiza el procedimiento bajo anestesia general en abril de 2012, mediante la técnica descrita por Marx. A la paciente se le colocan cuatro implantes de oseointegración en el sector anterior, a los 7 días postoperatorios se observa una adecuada posición de implantes, sin ninguna evidencia imagenológica de alteraciones (figura 10). 


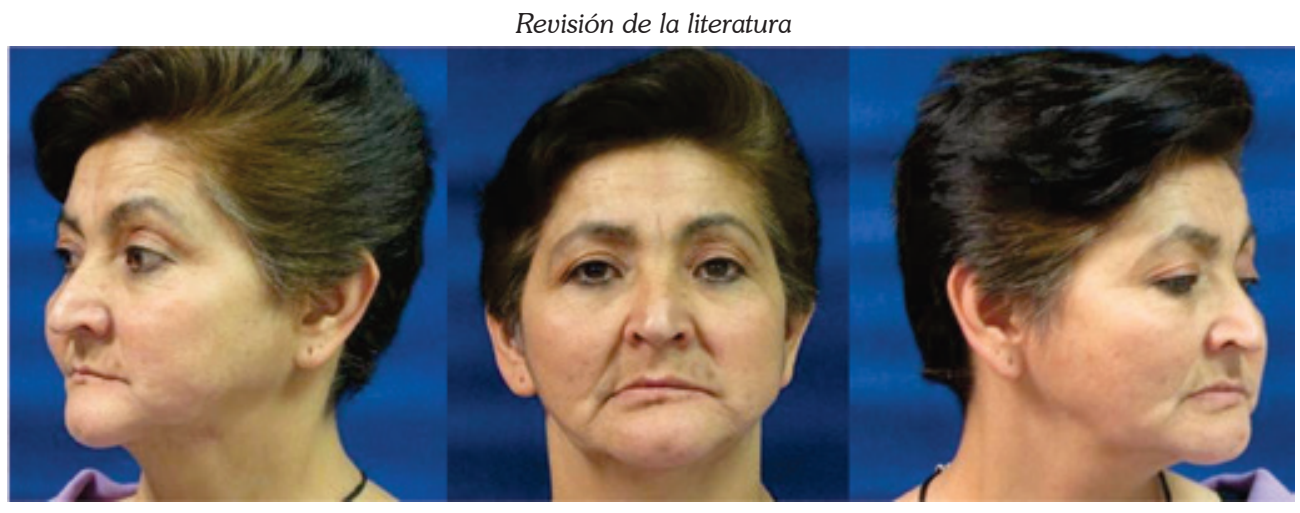

Figura 9. Caso Clínico \#3. Pérdida de soporte labial, ptosis de punta nasal y comisuras labiales, disminución de dimensión vertical a expensas del tercio inferior. Pseudoprognatismo mandibular.

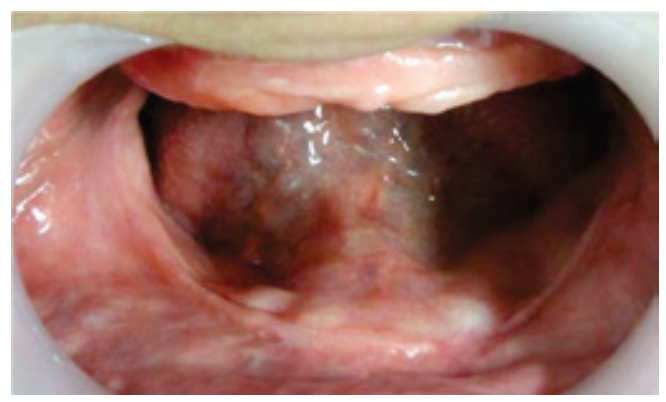

Figura 10. Reborde alveolar mandibular con reabsorción severa.
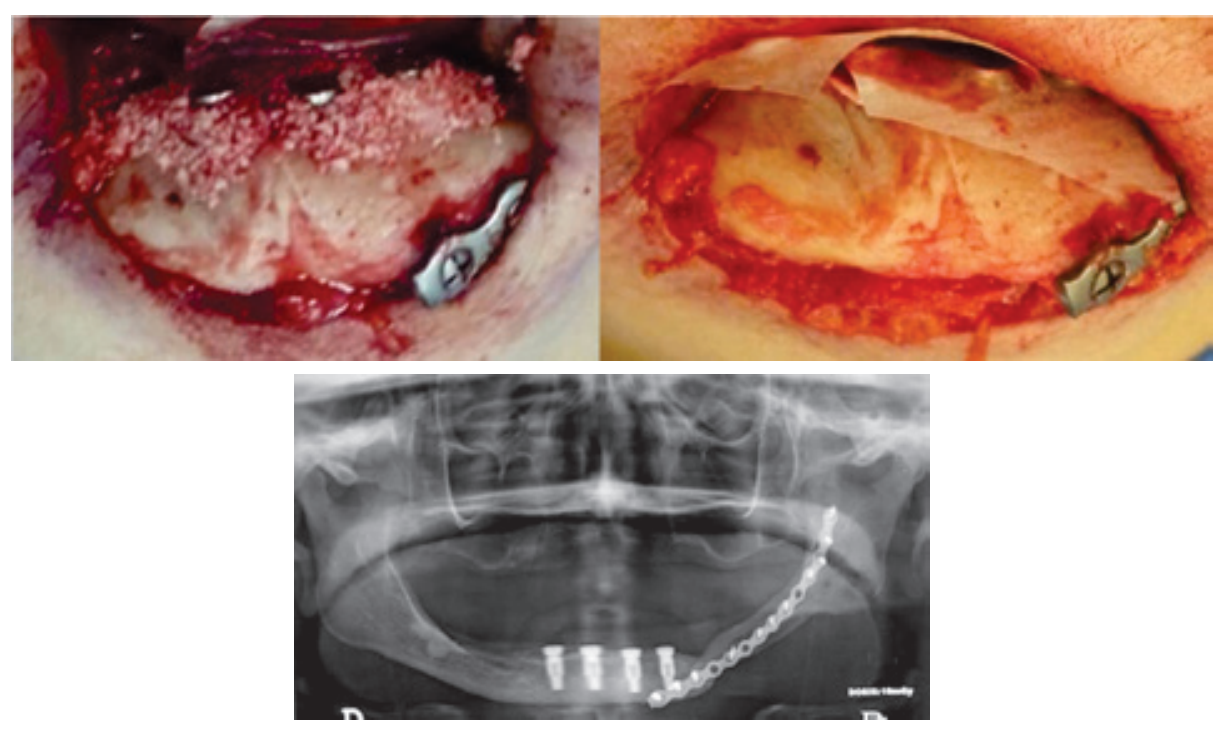

Figura 11. Colocación de 4 implantes de oseointegraciónde 3,75 mm de diámetro por $13 \mathrm{~mm}$ de longitud, injerto óseo autólogo corticomedular de cresta ilíaca anterior y membrana de colágeno para regeneración ósea guiada. 


\section{Discusión}

La técnica descrita por Marx y colaboradores en el año 2002, constituye un método novedoso que permite reconstruir la mandíbula con reabsorción severa, mediante la expansión de matriz de tejido blando, que en este caso corresponde a la mucosa del reborde residual. Es un procedimiento que tiene múltiples ventajas, entre ellas: es un método extraoral que evita la contaminación del injerto con el medio oral, supone la colocación de implantes de oseointegración en un solo tiempo quirúrgico, incrementa la resistencia del hueso mandibular, provee de buena retención a la rehabilitación implantosoportada y ganancia de altura ósea estable a largo plazo.

Es importante hacer énfasis en que la rehabilitación implantosoportada y la carga protésica sobre el injerto óseo incrementa el contenido mineral, lo cual es denominado "modelado positivo". El modelado positivo es evidente imagenológicamente a través del aumento de radiopacidad en una imagen panorámica y/o periapical asociada al implante. La redistribución de las cargas protésicas permite un proceso constante de modelado, y el tejido blando que rodea el hueso sirve de guía para el remodelado (aposición y reabsorción) del hueso que se encuentra dentro de sus confines.

Fisiológicamente, la reabsorción mandibular tiene sus inicios en largos períodos de enfermedad periodontal no tratada, seguido de la pérdida dental, reabsorción ósea, y por ende disminución del volumen de tejido queratinizado que envuelve el reborde alveolar. Con el transcurso de los años, la compresión protésica o alimentaria (en el caso de los pacientes que no utilizan prótesis) incrementa la inflamación, fibrosis y contracción volumétrica de la mucosa del reborde alveolar. La contracción del volumen de tejido blando exacerba la pérdida del volumen óseo subyacente de manera directa.

Otro factor a tomar en consideración, es la edad y el género del paciente a tratar. El 95\% de las mujeres de edad avanzada, postmenopáusicas, afectadas por la reabsorción mandibular severa, presentan disminución de los niveles de estrógenos, que se traduce en remodelado y modelado negativos (pérdida de volumen y densidad ósea respectivamente) (1). Por lo cual se sugiere, control estricto de la condición hormonal de las pacientes que se encuentran en esta etapa de la vida.
Marx encontró en los pacientes sometidos a esta técnica, que la reabsorción del injerto óseo se daba únicamente hasta el nivel de los implantes de oseointegración, lo cual sustenta el hecho de que mantener un espacio subperióstico evita la contracción del tejido blando sobre el injerto, evitando su reabsorción (1).

La técnica "Tent Pole" también ha sido implementada para tratar defectos marginales producto de cirugía ablativa por procesos tumorales. Camichael y cols. (15) describieron el uso de esta técnica para la reconstrucción del cuerpo mandibular posterior a resección de un osteosarcoma. Fenton y cols. (16) la utilizaron para la reconstrucción de un defecto mandibular posterior a la resección de un retinoblastoma metastásico, en un paciente pediátrico quien había sido tratado mediante un colgajo de peroné. Wannfors y cols. (17) reportaron la técnica en una paciente con osteogénesis imperfecta tipo III con agenesia de los dientes anteroinferiores con resultados satisfactorios. Siguiendo el protocolo de Marx, realizaron el aumento de reborde residual en el sector anteroinferior mandibular. Colocaron injerto óseo corticomedular obtenido de la cresta ilíaca anterior del paciente, en combinación con plasma rico en plaquetas.

El papel que juega el plasma rico en plaquetas (PRP) en las técnicas de aumento óseo ha sido investigado en varios reportes en la literatura, algunos de esos estudios afirman que el PRP favorece la cicatrización ósea por sus propiedades osteogénica y osteoinductiva (18-24), mientras que otros estudios no lo sustentan (25-28).

Korpi y cols. (29) aplicaron la técnica descrita por Marx, excluyendo la colocación de plasma rico en plaquetas, en 22 pacientes ( 3 hombres, 19 mujeres) con antecedente de fracaso del manejo protésico convencional. Colocaron cuatro implantes de oseointegración, en la zona anterior mandibular por la vía submental y cubiertos con injerto óseo corticomedular de cresta ilíaca posterior. El seguimiento de los casos varió desde 3 hasta 9 años postoperatorios. El curso postoperatorio de todos los casos resultó sin complicaciones. La densidad radiográfica del injerto, en la mayoría de los casos, igualó a la densidad radiográfica del hueso nativo a los 3 meses postoperatorios. Obtuvieron un aumento alveolar promedio de $6,3 \mathrm{~mm}$, sin evidencia radiográfica de reabsorción ósea periimplantaria en el seguimiento a largo plazo. 
Según los resultados de ese estudio es posible suponer que la técnica "Tent Pole" puede ser realizada de manera segura sin la adición de PRP, sería necesario establecer un grupo control, para determinar si existen diferencias significativas entre la modificación de la técnica y la propuesta por Marx.

Este artículo reporta una serie heterogénea de casos, siendo la segunda publicación en donde se presenta un paciente manejado mediante la técnica "Tent Pole" en un colgajo microvascular de peroné con resultados satisfactorios. La primera paciente de esta serie, con antecedente de Esclerodermia, siendo una enfermedad autoinmune que afecta la piel, órganos, y tejido conectivo en general. Se caracteriza por endurecimiento de la piel y el tejido conectivo, y en algunos casos alteraciones vasculares.

Las manifestaciones orales incluyen neuropatía del trigémino, xerostomía, ensanchamiento del ligamiento periodontal, microstomía y reabsorción dental idiopática. La xerostomía es común, debido a la fibrosis del ducto salivar que reduce el flujo, lo que trae como consecuencia falla en el sistema de equilibrio del $\mathrm{pH}$ en la cavidad oral, aumentado el riesgo de caries y enfermedad periodontal. Existen una serie de cambios en el tejido conectivo, que produce una reducción en la vascularidad de la mucosa oral, ulceraciones, y fibrosis (30-33). La fibrosis a su vez, produce constricción sobre el hueso mandibular, ocasionando reabsorción marcada en el reborde alveolar edéntulo y recesión gingival en pacientes dentados, aunque la reabsorción mandibular en estos pacientes, a pesar de ocurrir rápidamente, es asintomática. Sin embargo, la reabsorción mandibular severa en estos pacientes incrementa el riesgo de desarrollar neuropatía trigeminal, fracturas patológicas y osteomielitis (34-37).

El tercer caso, una paciente con síndrome de Ehler Danlos, que corresponde a un grupo heterogéneo de desórdenes caracterizado por hiperelasticidad cutánea, hipermovilidad articular, susceptibilidad a sufrir equimosis, alteración de la cicatrización, la mayoría de las veces asociadas a anomalías en la información genética destinada a la síntesis de colágenos fibrilares (38). Aunque no se ha reportado relación directa del síndrome con fracasos en la oseointegración de implantes dentales, sí se ha observado mayor riesgo de osteoporosis (39).

\section{Conclusiones}

El reborde alveolar mandibular con reabsorción severa ( $<6 \mathrm{~mm}$ de altura) puede ser reconstruido con una ganancia de hasta $15 \mathrm{~mm}$ de altura. Este abordaje quirúrgico permite expandir el volumen de tejidos blandos utilizando los implantes como medio de suspensión. El espacio creado permite entonces la consolidación del injerto, evitando la influencia negativa de la contracción de los tejidos sobre el mismo. Ésto favorece el modelado positivo (aumento de densidad ósea) una vez se cargan los implantes, conservándose la altura ganada a largo plazo (1). En esta serie de casos se corrobora la versatilidad de la técnica descrita por Marx. Es de gran utilidad en el manejo de pacientes con secuelas de trauma en donde las opciones de manejo son limitadas y en aquellos con condiciones sistémicas que podrían comprometer los resultados.

Es una técnica que promete reducir la tasa de complicaciones y los costos hospitalarios que supone el manejo de dichas complicaciones. Es una opción más de reconstrucción mandibular y rehabilitación para pacientes con pronóstico protésico reservado.

\section{Referencias}

1. Marx R, Shellenberger T, Wimsatt J. Severely Resorbed Mandible: Predictable Reconstruction With Soft Tissue Matrix Expansion (Tent Pole) Grafts. J Oral Maxillofac Surg. 2002; 60: 878-888.

2. Bell R, Blakey G, White R, Dennis G. Hillebrand and Anthony Molina, Staged Reconstruction of the Severely Atrophic Mandible With Autogenous Bone Graft and Endosteal Implants. J Oral Maxillofac Surg. 2002; 60: 1135-1141.

3. Le B, Rohrer M, Prasad HS. Screw "tent-pole" grafting technique for reconstruction of large vertical alveolar ridge defects using human mineralized allograft for implant site preparation. J Oral Maxillofac Surg. 2010; 68(2): 428-35.

4. Manfro R, Batassini F, Bortoluzzi M. Severely resorbed mandible treated by soft tissue matrix expansion (tent pole) grafts: Case Report. Implant Dent. 2008; 17(4): 408-13.

5. Copel M. Spontaneous fracture of an atrophic edentulous mandible treated without fixation. Br J Oral Surg. 1982; 20: 22.

6. Lypka M, Yarmand D, Burstein J, Tso V, Yamashita D. Dental Implant Reconstruction in a patient with Ectoderma Dysplasia Using Multiple bone Grafting Techniques. J Oral Maxillofac Surg. 2008; 66: 1241-1244

7. Baker R, Terry B, Davis W. Long term results ofalveolar ridge augmentations. J Oral Surg. 1979; 37: 486.

8. Davis WH, Martinoff JT, Kaminski NM. Long-term follow-up oftransoral rib grafts for mandibular atrophy. J Oral Maxillofac Surg. 1984; 42: 606.

9. Stoelinga P, de Koomen H, Tideman H. A reappraisal of the interposed bone graft augmentations of the atrophicmandible. J Maxillofac. 1983; Surg. 11: 107. 


\section{COLOCACIÓN DE IMPLANTES DE OSEOINTEGRACIÓN EN MANDÍBULAS CON REABSORCIÓN SEVERA UTILIZANDO LA TÉCNICA \\ "TENT POLE". SERIE DE CASOS}

10. Harle F. Visor osteotomy to increase the absolute height of theatrophied mandible. J Maxillofac Surg. 1975; 3: 257.

11. Louis P, Gutta R, Said-Al-Naief N. Reconstruction of the maxilla and mandible with particulate bone graft and titanium mesh for implant placement. J Oral Maxillofac Surg. 2008; 66: 235.

12. Simion M, Jovanovic S, Tinti C. Long term evaluation of osseointegrated implants inserted at the same time or after vertical ridge augmentation: A Retrospective Study on 123 implants with 1-5 year follow up. Clin Oral Implants Res. 2001; 12: 35.

13. Block M, Degen M. Horizontal ridge augmentation using human mineralized particulate bone: Preliminary reults. J Oral Maxillofac Surg. 2004; 62: 67.

14. Eyrich G, Grätz K, Sailer H. Surgical Treatment of Fractures of the edentulous mandible. J Oral Maxillofac Surg. 1997; 55: 1081.

15. Carmichael R, Sándor G, Bilko S. Reconstruction of anablative defect of the mandible in a young man using lateral soft tissue matrix expansion grafting and a novel technique for construction of a retrievable fixed prosthesis. Forum Implantol. 2008; 4: 1.

16. Fenton CC, Nish IA, Carmichael RP, Sàndor GK. Metastatic mandibular retinoblastoma in a child reconstructed with soft tissue matrix expansion grafting. J Oral Maxillofac Surg. 2007; 65: 11.

17. Wannfors K, Johansson C, Donath K, Mult D. Augmentation of the mandible via a Tent Pole Procedure and Implant Treatment in a Patient with Type III Osteogenesis mperfecta: Clinical and histologic Considerations. Int J Oral Maxillofac Implants. 2009; 24: 1144-1148.

18. Marx R, Carlson E, Eichstaedt R. Platelet-rich plasma: Growth factor enhancement for bone grafts. Oral Surg Oral Med Oral Pathol Oral Radiol Endod. 1998; 85: 6.

19. Merkx M, Fennis J, Verhagen C. Reconstruction of themandible using preshaped $2.3 \mathrm{~mm}$ titanium plates, autogenousparticulate cortico-cancellous bone grafts and platelet rich plasma: A report on eight patients. Int J Oral Maxillofac Surg. 2004; 33: 733.

20. Mooren R, Merkx M, Kessler P. Reconstruction of themandible using preshaped 2.3-mm titanium plates, autogenouscortical bone plates, particulate cancellous bone, and plateletrichplasma: A retrospective analysis of 20 patients. J Oral Maxillofac Surg. 2010; 68: 10.

21. Schlegel K, Kloss F, Kessler P. Bone conditioning toenhance implant osseointegration: An experimental study inpigs. Int $\mathrm{J}$ Oral Maxillofac Implants. 2003; 18: 4.

22. Simon E, Merkx M, Shubi F. Reconstruction of the mandible after ablative surgery for the treatment of aggressive benign odontogenic tumors in Tanzania: A preliminary study. Int J Oral Maxillofac Surg. 2006; 35: 5.

23. Thorn J, Sorensen H, Weis-Fogh U. Autologous fibrin glue withgrowth factors in reconstructive maxillofacial surgery. Int $J$ Oral Maxillofac Surg. 2004; 33: 95.
24. Zechner W, Tangl S, Tepper G, Fürst G, Bernhart T, Haas R, et al. Influence of platelet-richplasma on osseous healing of dental implants: A histologic anhistomorphometric study in minipigs. Int J Oral Maxillofac Implants. 2003; 18:1.

25. Aghaloo T, Moy P, Freymiller E. Investigation of plateletrichplasma in rabbit cranial defects: A pilot study. J Oral Maxillofac Surg. 2002; 60: 10.

26. Gerard D, Carlson ER, Gotcher JE, Jacobs M. Effects of platelet-richplasma on the healing of autologous bone grafted mandibulardefects in dogs. J Oral Maxillofac Surg. 2006; 64: 3.

27. Jakse N, Tangl S, Gilli R, Berghold A, Lorenzoni M, Eskici A, et al. Influence of PRP on autogenoussinus grafts: An experimental study on sheep. Clin Oral Implants Res. 2003; 14: 5.

28. Schlegel K, Donath K, Rupprecht S. De novo bone formation using bovinecollagenand platelet-rich plasma. Biomaterials. 2004; 25: 5387.

29. Korpi J, Kainulainen V, Sándor G, Oiknarinen K. Long-Term Follow-Up of SeverelyResorbed Mandibles Reconstructed Using Tent Pole Technique WithoutPlatelet-Rich Plasma. J Oral Maxillofac Surg. 2012; 70: 2543-2548.

30. Fischer D, Patton L. Scleroderma: oral manifestations and treatment challenges. Spec Care Dentist. 2000; 20(6): 240-4.

31. Rout P, Hamburger, J, Potts, A. Orofacial radiological manifestations of systemic sclerosis. Dentomaxillofac Rad. 1996; 25(4): 193-6.

32. Spackman G. Scleroderma: what the general dentist should know. Gen Dent. 1999; 47(6): 576-9.

33. Wood R, Lee P. Analysis of the oral manifestations of systemic sclerosis (scleroderma). Oral Surg, Oral Med, Oral Pathol. 1988; 65(2): 172-8.

34. Iannello S, Camuto M., Cantarella S, Cavaleri A, Ferriero P, Leanza A, et al. Rheumatoid syndrome associated with lung interstitial disorder in a dental technician exposed to ceramic silica dust. A case report and critical literature review. Clin Rheum. 2002; 21(1): 76-81.

35. Tolle S. Scleroderma: considerations for dental hygienists. Int $\mathrm{J}$ Dent Hyg. 2008; 6(2): 77-83.

36. Jagger R, Absi E, Jeremiah H, Sugar A. Bilateral mandibular condylisis in a patient with systemic sclerosis. Dentomaxillofac Rad. 2006; 35: 461-463.

37. Auluck A, Pai K, Shetty C, Shenoi S. Mandibular resorption in progressive systemic sclerosis: a report of three cases. Dentomaxillofac Rad. 2005; 34: 384-386.

38. Nuytinck L, Freund M, Lagae L, Pierard G, Hermanns-Le T, De Paepe A. Classical Ehlers-Danlos Syndrome Caused by a Mutation in Type I Collagen. Am. J. Hum. Genet. 2000; 66: 1398-1402.

39. Theodorou S, Theodorou D, Kakitsubata Y, Adams J. Low Bone Mass in Ehlers-Danlos Syndrome. Intern Med. 2012; 51: 3225-3226. 